УДК 004.8:796

Мельников А. Ю., Кадацкий Н. А.

\title{
РАЗРАБОТКА ИНФОРМАЦИОННОЙ СИСТЕМЫ ДЛЯ ПРИБЛИЗИТЕЛЬНОГО НАХОЖДЕНИЯ ПОКАЗАТЕЛЕЙ СПОРТСМЕНА-МЕТАТЕЛЯ ПРИ ПОМОЩИ МАТЕМАТИЧЕСКОГО МОДЕЛИРОВАНИЯ ТОЛКАНИЯ ЯДРА И ПРИМЕНЕНИЯ НЕЙРОСЕТЕВЫХ ТЕХНОЛОГИЙ
}

Современный уровень развития легкой атлетики, в частности - толкания ядра, ставит задачу разработки новых, более рациональных средств и методов спортивной подготовки, которые содействуют быстрому и надежному достижению высоких спортивных результатов. Особенно возросла необходимость в научных исследованиях после роста спортивных достижений, объясняющегося, в первую очередь, хорошей силовой подготовкой метателя. Однако силу нельзя увеличивать бесконечно, и дальнейший рост результатов возможен за счет лучшего сочетания других параметров.

Объектом исследования является математическое описание процесса метания ядра. Предметом исследования выступает поиск оптимального сочетания параметров (показателей) спортсмена-метателя для достижения максимальной дальности броска.

Согласно [1], дальность полета ядра L зависит от таких величин:

- начальной скорости $\mathrm{V}_{0}$, с которой ядро выталкивается;

- угла к горизонту $\theta_{0}$, под которым ядро выталкивается;

- высоты над землей $\mathrm{H}_{0}$, на которой ядро покидает руку (рис. 1).

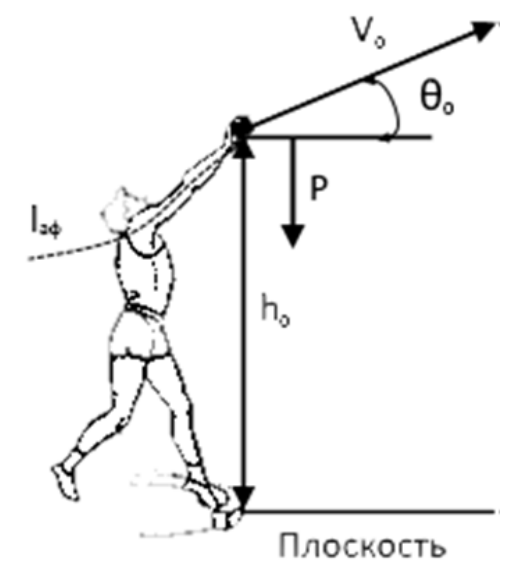

Рис. 1. Изображение вектора физических переменных

$$
\mathrm{L}=\frac{21 \mathrm{~F}_{\mathrm{r}}}{\mathrm{P}} \cos \omega_{0}\left(\sin \omega_{0}+\sqrt{\sin ^{2} \omega_{0}+\frac{P h_{0}}{l_{a \phi} F_{\mathrm{r}}}}\right)
$$

Из формулы (1) видно, что чем меньше сила воздействия на ядро, тем большим должен быть угол направления этой силы, который демонстрирует рис.1. При определенном для данной силы угле наступает оптимальное сочетание всех величин, что приводит к максимальной дальности полета снаряда.

Для проведения расчетов по формулам из [1] была спроектирована информационная модель системы на унифицированном языке моделирования UML [2]. Функциональные возможности системы представлены в виде диаграммы вариантов использования (рис. 2). 


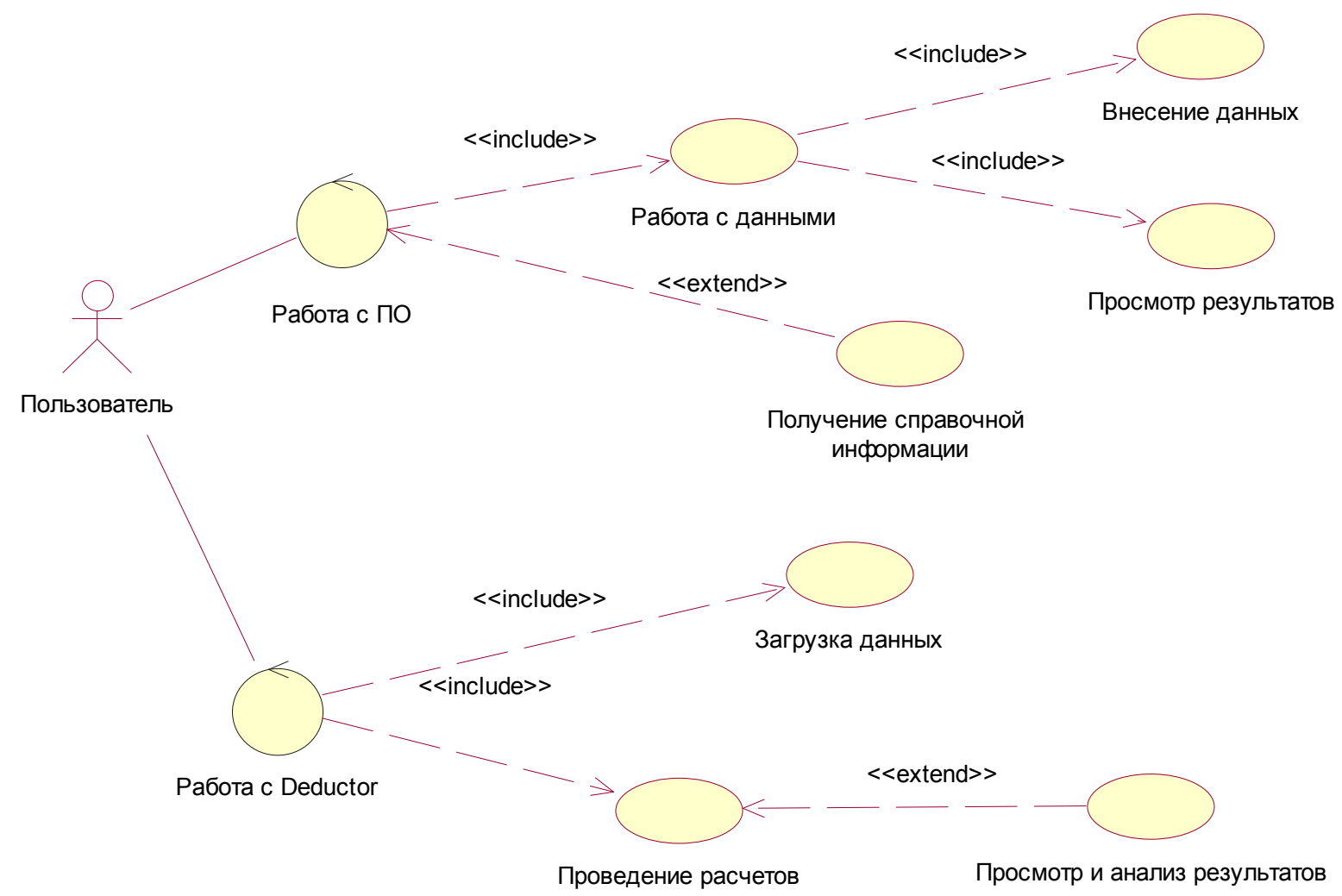

Рис. 2. Диаграмма вариантов использования информационной системы для приблизительного нахождения показателей спортсмена-метателя ядра

Модель была реализована в виде приложения [3-4], позволяющего провести моделирование толкания ядра с места и определить оптимальное сочетание показателей для определенного ядра.

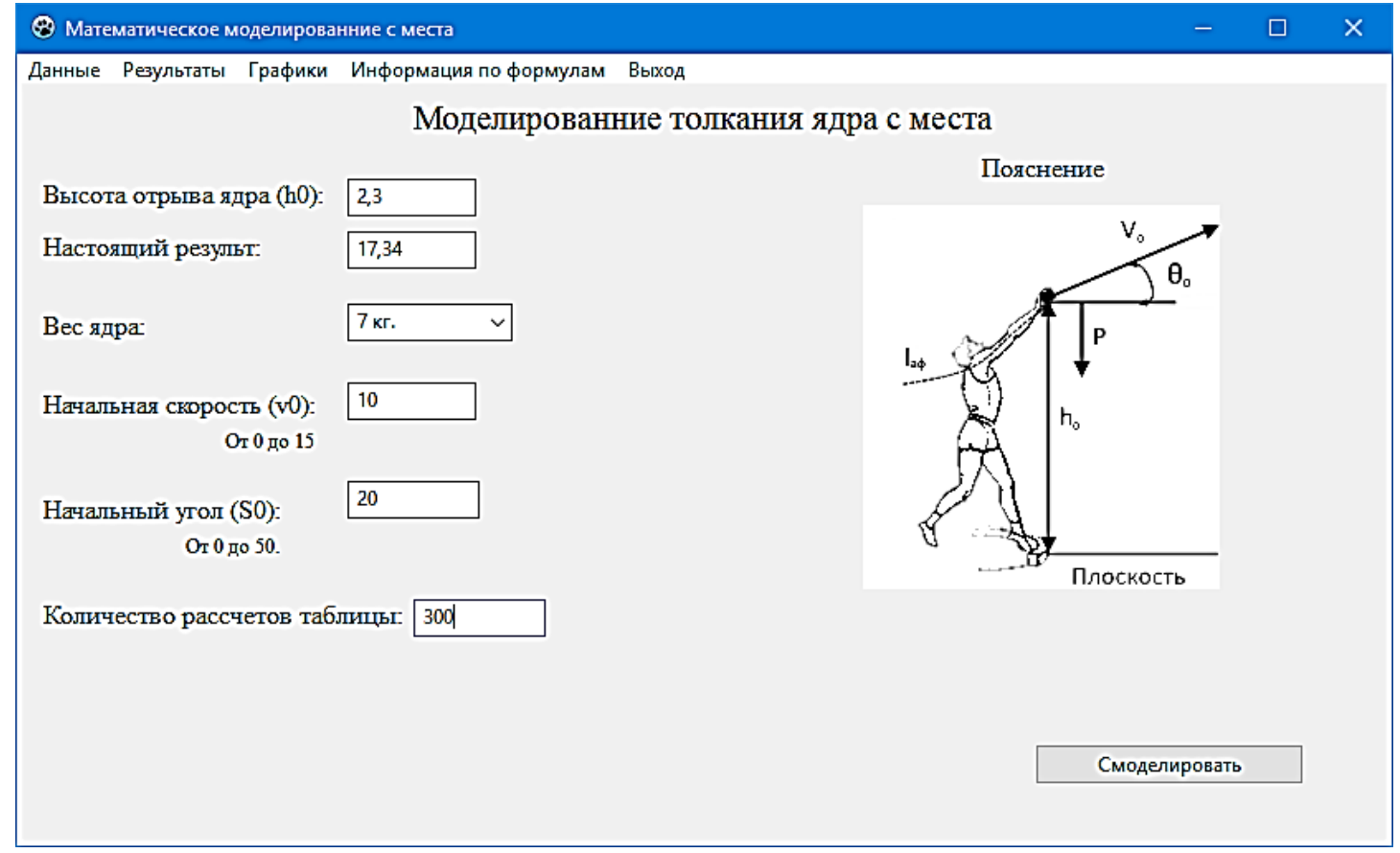

Рис. 3. Основная вкладка приложения (начальные данные для 7 кг ядра) 
В основной вкладке «Данные» пользователь вводит нужные показания для расчета: высоту отрыва ядра, настоящий результат (по которому идет приблизительный поиск), массу ядра, начальный угол (от которого будет начинаться поиск и все расчеты), начальную скорость (с которой будет начат циклический процесс моделирования), количество расчетов в таблице (которое будет записано в таблицу для сравнения и получения информации). Основной величиной, от которой зависит скорость вылета снаряда, а значит и результат метания, является сила, скорость и угол, под которым спортсмен воздействует на снаряд.

После моделирования на вкладке «Результаты» мы можем увидеть таблицу со всеми рассчитанными программой углами и их отношения к скорости выпуска ядра. Для более точного результата проведем несколько приближенных поисков.


Рис. 4. Приближенный поиск толкателя 7 кг и 6 кг ядра

Данные, которые мы ввели в программу, показали следующий результат. Спортсмен, который толкает ядро массой 7 кг, приблизительно выталкивает его под углом 36 градусов, со скоростью 12,3 метра в секунду. Сила разгона снаряда при этом составляет 363,95 килограмма. А спортсмен, толкающий ядро массой 6 килограмм на 20 метров 13 сантиметров, должен выпускать ядро под скоростью 13,4 м/сек с углом выпуска 38 градусов и силой 359,12 килограмм.

Очевидно, что описание спортивной техники исключительно уравнениями механики может не учитывать ряд факторов, которые, являясь малозначимыми для абсолютных значений результатов, могут оказать серьезное влияние на относительные показатели.

В физической культуре и спорте нейронные сети используются для анализа и прогнозирования показателей физической подготовленности спортсменов, а также результатов спортивных соревнований. Эффективность использования нейронных сетей объясняется возможностью моделирования физиологических процессов в организме человека, носящих нелинейный характер, а также способностью нейронных сетей к самообучению [5-6].

В [7] приводятся данные о характеристиках восьми спортсменов (возраст, рост, масса тела, предпочитаемый метод метания), а также их спортивные результаты (начальная скорость полета ядра, угол метания, высота отрыва от руки и расстояние полета).

\begin{tabular}{|c|c|c|c|c|c|c|c|c|}
\hline Name & Technique & $\begin{array}{c}\text { Age } \\
\text { [years] }\end{array}$ & $\begin{array}{c}\text { Helght } \\
{[\mathrm{m}]}\end{array}$ & $\begin{array}{c}\text { Weight } \\
{[\mathrm{kg}]}\end{array}$ & $\begin{array}{c}\mathrm{vO} \\
{[\mathrm{m} / \mathrm{s}]}\end{array}$ & $\begin{array}{l}\text { av } \\
\left.{ }^{\circ}\right]\end{array}$ & $\begin{array}{l}\mathrm{HO} \\
{[\mathrm{m}]}\end{array}$ & $\begin{array}{l}\text { Distanc } \\
{[\mathrm{m}]}\end{array}$ \\
\hline Can & Rotational & 28 & 1,98 & 140 & 14 & 37,8 & 2,29 & . \\
\hline Maj & 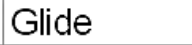 & 27 & 04 & 132 & 13,8 & 39,3 & 2,43 & 1 \\
\hline Barte & & 31 & 1,87 & 135 & 14 & 33,6 & 2,12 & \\
\hline Hoffa & & 31 & 1,8 & 133 & 14 & 34,4 & 2,0 & \\
\hline Nels & & 34 & 1,83 & 115 & 14,1 & 32,9 & 2,0 & \\
\hline Lyzh & & 28 & 1,89 & 110 & 13,6 & 39,2 & 2,22 & \\
\hline Mikhnevic & Glide & 33 & 2,02 & 127 & 13,4 & 37,7 & 2,43 & 0,7 \\
\hline /odovr & otat & 31 & 96 & 145 & 3,7 & 3,1 & 2,25 & \\
\hline
\end{tabular}

Рис. 5. Данные об атлетах 
С математической точки зрения можно сформулировать две задачи прогнозирования:

- по имеющимся данным о возрасте, росте, массе тела атлета, а также характеристиках полета ядра определить дальность этого полета;

- по имеющимся данным о возрасте, росте, массе тела атлета, а также дальности полета ядра определить оптимальное сочетание характеристиках полета - начальной скорости, угле и высоте отрыва.

Обе задачи можно решить методами искусственных нейронных сетей. В качестве модели нейронной сети целесообразно выбрать двухслойный персептрон. Оценка предпочтительного числа нейронов в скрытом слое проведем, используя известное неравенство [8-10]. В первом случае величину скрытого слоя примем равным 3 нейронам, во втором - 5. Тип активационной функции - сигмоида. Обучение сети проводится методом обратного распространения ошибок. Расчет был проведен в среде Deductor Studio [11].

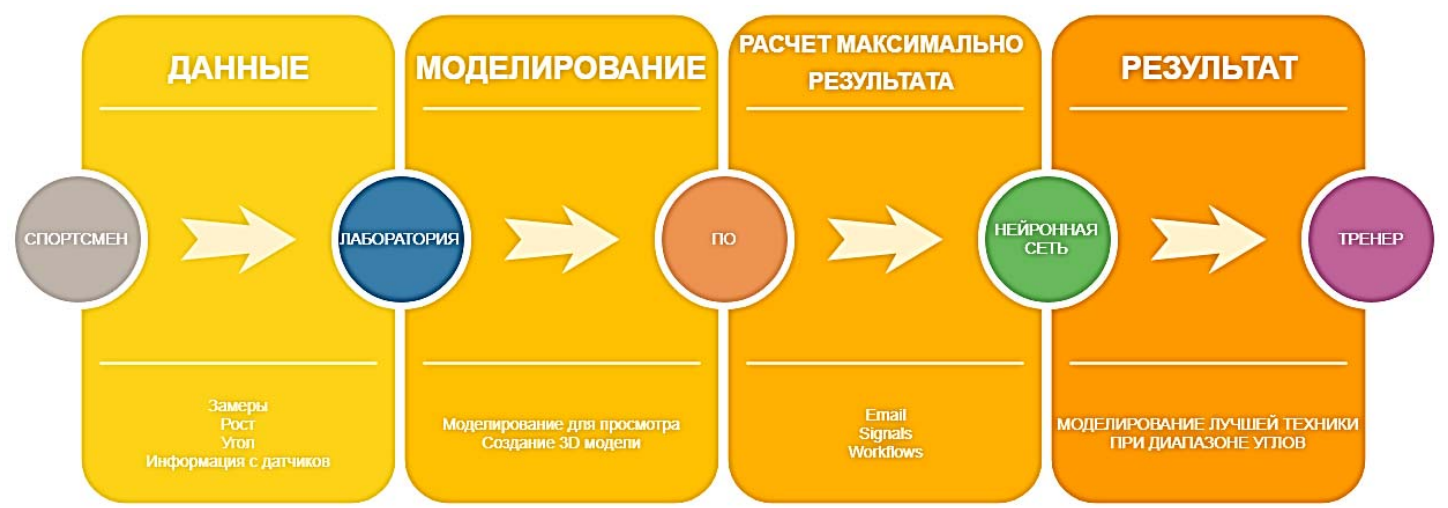

Рис. 6. Структура всей системы моделирования показателей
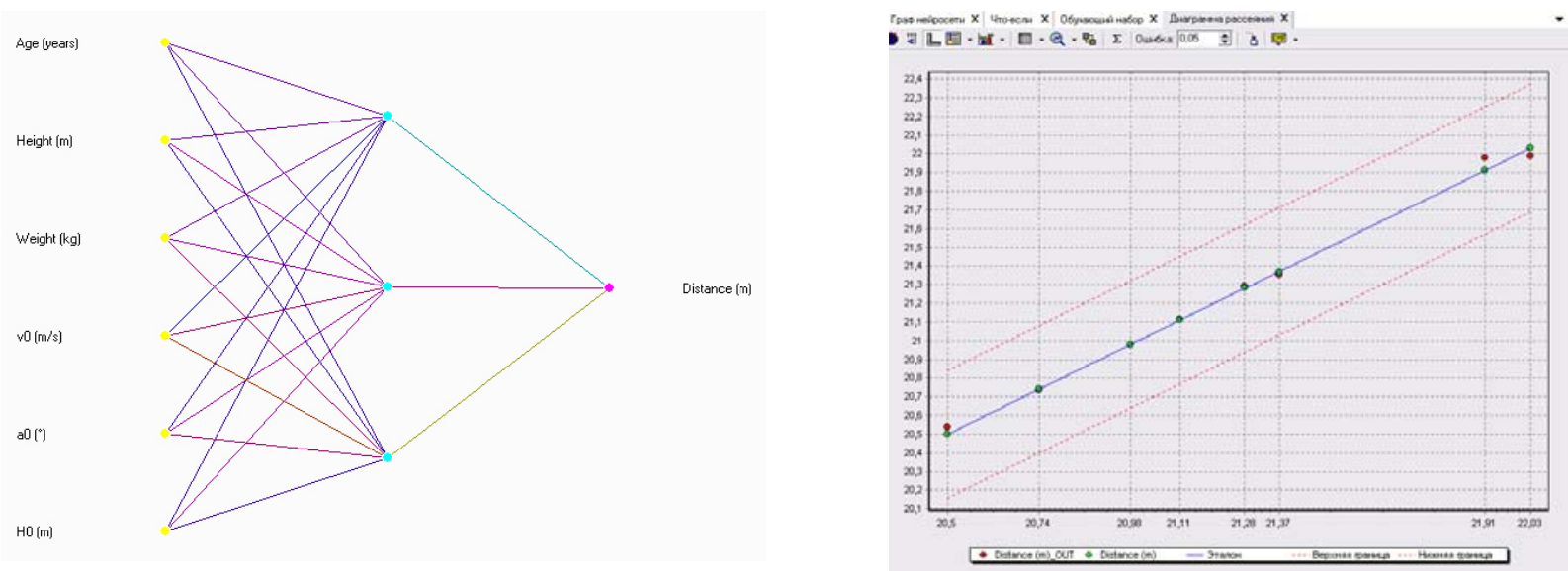

Рис. 7. Граф и диаграмма рассеяния нейронной сети MLP-6-3-1


Рис. 8. Граф и диаграмма рассеяния нейронной сети MLP-4-5-3 


\section{ВЫВОДЫ}

В статье представлено описание информационной системы, которая позволяет рассчитать оптимальное сочетание скорости, угла выпуска ядра и силы, с которой спортсмен должен толкать ядро, для достижения максимальной дальности полета. В то же время сделан вывод, что описание спортивной техники исключительно уравнениями механики может не учитывать ряд факторов, которые, являясь малозначимыми для абсолютных значений результатов, могут оказать серьезное влияние на относительные показатели, и приведены примеры использования метода искусственных нейронных сетей в среде Deductor Studio Lite для решения такой задачи. В примерах можно по имеющимся данным о возрасте, росте, массе тела атлета, а также характеристикам полета ядра определить дальность этого полета; с другой стороны - по имеющимся данным о возрасте, росте, массе тела атлета, а также дальности полета ядра определить оптимальное сочетание характеристик полета - начальной скорости, угла и высоте отрыва.

\section{СПИСОК ИСПОЛЬЗОВАННОЙ ЛИТЕРАТУРЫ}

1. Тутевич В. Н. Теория спортивных метаний / В. Н. Тутевич. - Москва, 1956. - 310 с.

2. Мельников А. Ю. Объектно-ориентированный анализ и проектирование информационных систем: учебное пособие / А. Ю. Мельников. - Изд. 2-е, перераб. и доп. - Краматорск : ДГМА, 2013. - 172 с.

3. Кадацкий Н. А. Приблизительное нахождение показателей спортсмена-метателя при помощи математического моделирования толкания ядра и программного обеспечения собственной разработки / А. Ю. Мельников, Н. А. Кадацкий // Молодежь в науке: Новые аргументы:: Сборник научных работ VIII-го Международного молодежного конкурса (Россия, г. Липеик, 30 марта 2018 г.). Часть I / Отв. ред. А. В. Горбенко. - Липеик : Научное партнерство «Аргумент», 2018. - C. 66-70.

4. Kadatsky N. A. On the use of mathematical modeling for the approximate finding of indicators athlete core thrower / O. Yu. Melnykov, N. A. Kadatsky // Матеріали міжнародної науково-практичної конференції «Universuт Viеw 6». - Вінниия : ТОВ «Нілан-ЛТД», 2018. - С. 293-294.

5. Касюк С. Т. Использование нейронных сетей для анализа и прогнозирования данных в физической культуре и спорте / С. Т. Касюк, Е. М Вахтомова // Научно-теоретический журнал «Ученые записки». - 2013. № 12 (106). - C. 72-77.

6. Крутиков А. К. Прогнозирование спортивных результатов в индивидуальных видах спорта с помощьью обобщенно-регрессионной нейронной сети / А. К. Крутиков // Молодой ученьй. - 2018. - № 12. - C. 22-26. URL: https://moluch.ru/archive/198/48884.

7. Wilko Schaa. Biomechanical Analysis of the Shot Put at the 2009 IAAF World Championships in Athletics / Schaa Wilko. - New Studies in Athletics, № 3-4, 2010. - C. 9-21. - URL: https://www.researchgate.net/publication/265661202.

8. Каллан Р. Основные конщепции нейронных сетей / Р. Каллан. - М. : Вильямс, 2001. - 288 с.

9. Хайкин, Саймон. Нейронные сети: полный курс, 2-е издание : пер. с англ. - М. : Издательский дом Вильямс, 2006. - 1104 с.

10. Ковалевский С. В. Создание и применение нейронных сетей для решения прикладных задач : Учебнометодическое пособие для студентов специильности «Интеллектуальные системы принятия решений» / С. В. Ковалевский, В. Б. Гитис. - Краматорск : ДГМА, 2008. - 75 с.

11. BaseGroup Labs: официальный сайт. - URL: https://basegroup.ru/community/articles/intro (05.03.2019). 